\title{
Fixed Point for Weakly Compatible Maps Satisfying Generalized Contraction Principle
}

\author{
J. G. Mehta ${ }^{{ }^{*}}$ and M. L. Joshi ${ }^{2}$ \\ ${ }^{1}$ Harish Chandra Research Institute, Allahabad, India \\ ${ }^{2}$ Department of Mathematics, M \& N Virani Science College, Rajkot, India \\ Received 21 November 2010, accepted in final revised form 4 June 2011
}

\begin{abstract}
We prove common fixed point theorems for a pair of weakly compatible mappings satisfying a generalized contraction principle by using a control function and implicit relation. We also establish invariant approximation result as an application of the result.

Keywords: Common fixed point; Weakly compatible maps; Control function; Invariant approximation.

(C) 2011 JSR Publications. ISSN: 2070-0237 (Print); 2070-0245 (Online). All rights reserved.

doi:10.3329/jsr.v3i3.6476 J. Sci. Res. 3 (3), 539-545 (2011)
\end{abstract}

\section{Introduction.}

Sessa [1] introduced weakly commuted mappings which was generalized as compatible mappings by Jungck [2]. The notion of $R$-weakly commuting mappings was coined by Pant [3]. The term called weakly compatible mappings was defined by Jungck and Rhoades [4].

Generalization for weakly contractive mapping in Hilbert space was proved by several authors [5-9]. Generalization for weakly contractive mapping in complete metric space was proved by Rhoades [7].

Fixed point theorems for a self mapping by altering distances between the points and using a control function were proved by Park [10] and Khan et al. [11]. Sastry [8] extended the concept for weakly commuting pairs of self mappings and proved common fixed point theorem in a complete metric space by using the control function.

Dutta and Choudhury [6] obtained a fixed point result by generalizing the concept of control function and the weakly contractive mapping. Jungck [12] proved a common fixed point theorem for commuting mappings generalizing the Banach's contraction principle.

The existence of invariant approximation using fixed point theorem was established by Meinardus [13] which was generalized by Brosowski [14]. Subrahmanyam [15] and Singh

\footnotetext{
*Corresponding author: jgm_hri@yahoo.in
} 


\section{Fixed Point for Weakly Compatible Maps}

[16] relaxed the linearity of the mapping and the convexity of the set of best approximants. Also the existence of invariant approximation using fixed point theorem was generalized by several authors [17-21].

The main purpose of this paper is to obtain common fixed point for weakly compatible mappings satisfying a more general weak contractive condition using implicit relation. As an application we have established best approximation result.

\section{Preliminaries}

We recall the definitions and results that will be needed in the sequel.

Definition 2.1 A sequence $\left\{x_{n}\right\}$ in a metric space $(X, d)$ is said to be convergent to a point $x \in X$, denoted by $\lim _{n \rightarrow \infty} x_{n}=x$, if $\lim _{n \rightarrow \infty} d\left(x_{n}, x\right)=0$.

Definition 2.2 A sequence $\left\{x_{n}\right\}$ in a metric space $(X, d)$ is said to be Cauchy sequence if $\lim _{t \rightarrow \infty} d\left(x_{n}, x_{m}\right)=0$ for all $n, m>t$.

Definition 2.3 A metric space $(X, d)$ is said to be complete if every Cauchy sequence in $X$ is convergent.

Definition 2.4 Let $f$ and $T$ be two self-maps on a set $X$. Maps $f$ and $T$ are said to be commuting if $f T x=T f x$ for all $x \in X$.

Definition 2.5 Let $f$ and $T$ be self-maps on a set $X$. If $f x=T x$, for some $x$ in $X$ then $x$ is called coincidence point of $f$ and $T$.

Definition 2.6 Let $f$ and $T$ be two self-maps defined on a set $X$. Then $f$ and $T$ are said to be weakly compatible if they commute at coincidence points. That is, if $f u=T u$ for some $u \in X$, then $f T u=T f u$.

Proposition 2.1 Let $f$ and $T$ be weakly compatible self mappings of a set $X$. If $f$ and $T$ have a unique point of coincidence, that is, $w=f x=T x$, then $w$ is the unique common fixed point of $f$ and $T$.

Definition 2.7 Let $f$ and $T$ be self mappings of a nonempty subset $M$ of a metric space $X$. The mapping $T$ is called $f$-contraction mapping, if there exists a real number $0 \leq k<1$ such that $d(T x, T y) \leq k d(f x, f y)$ for all $x, y \in M$.

Definition 2.8 [11] A control function $\varphi$ is defined as $\varphi: R^{+} \rightarrow R^{+}$which is continuous at zero, monotonically increasing and $\varphi(t)=0$ if and only if $t=0$.

Definition 2.9 [5] A self mapping $T$ of a metric space $(X, d)$ is said to be weakly contractive with respect to a self mapping $f: X \rightarrow X$, if for each

$x, y \in X, d(T x, T y) \leq d(f x, f y)-\varphi(d(f x, f y))$, where $\varphi:[0, \infty) \rightarrow[0, \infty)$ is a continuous and non decreasing function such that $\varphi$ is positive on $(0, \infty), \varphi(0)=0$ and $\lim _{t \rightarrow \infty} \varphi(t)=\infty$

Proposition $2.2[6]$ Let $(X, d)$ be a complete metric space and $T: X \rightarrow X$ be a self mapping satisfying 


$$
\varphi(d(T x, T y)) \leq \varphi(d(f x, f y))-\phi(d(f x, f y))
$$

where $\varphi, \phi:[0, \infty) \rightarrow[0, \infty)$ are both continuous and monotonic increasing function with $\varphi(x)=0=\phi(x)$ if and only if $x=0$.

Then $T$ has a unique fixed point.

\section{Implicit relations}

Let $\mathbf{F}^{*}$ be the set of continuous functions $F\left(t_{1}, t_{2}, t_{3}, t_{4}\right):[0, \infty)^{4} \rightarrow[0, \infty)$ satisfying the following conditions:

$\left(F_{1}\right) F$ is non decreasing in variables $t_{1}$.

$\left(F_{2}\right)$ For $u \geq 0, v \geq 0 \quad F(u+v, 0, u, v) \leq u$

$\left(F_{3}\right) \quad F(u, u, 0,0) \leq u$ and $F(0, u, 0, u) \leq u, \forall u>0$.

\section{Main Result}

In this section we prove a common fixed point theorem for a pair of weakly compatible mappings in complete metric spaces by using a control function and implicit relation.

Theorem 3.1 Let $(X, d)$ be a complete metric space. Suppose that the mappings $T$ and $f$ are two self-maps of $X$ satisfying the following conditions:

(i) $T(X) \subseteq f(X)$.

(ii) $T(X)$ is complete subspace of $X$.

(iii) $\varphi(d(T x, T y)) \leq \varphi(M(x, y))-\phi(M(x, y))$

where $M(x, y)=F\{d(T x, f y), d(T y, f x), d(T x, f x), d(T y, f y)\}$

and $\varphi:[0, \infty) \rightarrow[0, \infty)$ is continuous and monotonic increasing function and $\phi:[0, \infty) \rightarrow[0, \infty)$ is continuous and monotonic decreasing function with $\varphi(x)=0=\phi(x)$ if and only if $\mathrm{x}=0$ and $F \in F^{*}$.

(iv) The pair $(T, f)$ is weakly compatible.

Then $f$ and $g$ have a unique common fixed point.

Proof : let $x_{0}$ be an arbitrary point of $X$.

Since $T(X) \subseteq f(X)$, we can choose $x_{n}$ and $x_{n+1}$ in $X$ such that,

$$
T x_{n}=f x_{n+1} n=0,1,2 \ldots
$$

By using (iii) we have,

$$
\begin{aligned}
& \varphi\left(d\left(T x_{n+1}, T x_{n}\right)\right) \leq \varphi\left(M\left(x_{n+1}, x_{n}\right)\right)-\phi\left(M\left(x_{n+1}, x_{n}\right)\right) \\
& \text { where } M\left(x_{n+1}, x_{n}\right)=F\left\{d\left(T x_{n+1}, f x_{n}\right), d\left(T x_{n}, f x_{n+1}\right), d\left(T x_{n+1}, f x_{n+1}\right), d\left(T x_{n}, f x_{n}\right)\right\} \\
& =F\left\{d\left(T x_{n+1}, T x_{n-1}\right), d\left(T x_{n}, T x_{n}\right), d\left(T x_{n+1}, T x_{n}\right), d\left(T x_{n}, T x_{n-1}\right)\right\} \\
& \leq F\left\{\left(d\left(T x_{n+1}, T x_{n}\right)+d\left(T x_{n}, T x_{n-1}\right)\right), 0, d\left(T x_{n+1}, T x_{n}\right), d\left(T x_{n}, T x_{n-1}\right)\right\}
\end{aligned}
$$




\section{Fixed Point for Weakly Compatible Maps}

Thus form $\left(F_{2}\right)$ we have, $M\left(x_{n+1}, x_{n}\right) \leq d\left(T x_{n}, T x_{n-1}\right)$

Thus from (1)

$$
\begin{aligned}
& \varphi\left(d\left(T x_{n+1}, T x_{n}\right)\right) \leq \varphi\left(d\left(T x_{n}, T x_{n-1}\right)\right)-\phi\left(d\left(T x_{n}, T x_{n-1}\right)\right) \\
& \varphi\left(d\left(T x_{n+1}, T x_{n}\right)\right) \leq \varphi\left(d\left(T x_{n}, T x_{n-1}\right)\right)
\end{aligned}
$$

Now $\varphi$ is monotonic increasing function. This implies that, the sequence $\left\{d\left(T x_{n+1}, T x_{n}\right)\right\}$ is monotonic decreasing.

Hence there exists a real number, say $r \geq 0$ such that $\lim _{n \rightarrow \infty} d\left(T x_{n+1}, T x_{n}\right)=r$.

Therefore as $n \rightarrow \infty$, equation (2) implies that

$$
\varphi(r) \leq \varphi(r)-\phi(r)
$$

So that $\phi(r) \leq 0$, which is possible only if $r=0$.

Thus

$$
\lim _{n \rightarrow \infty} d\left(T x_{n+1}, T x_{n}\right)=0
$$

Now we show that $\left\{T x_{n}\right\}$ is a Cauchy sequence.

Let if possible we assume that $\left\{T x_{n}\right\}$ is not a Cauchy sequence

Then there exists an $\varepsilon>0$ and subsequences $\left\{n_{i}\right\}$ and $\left\{m_{i}\right\}$ such that

$$
\begin{aligned}
& m_{i}<n_{i}<m_{i+1} \text { and } \\
& d\left(T x_{m_{i}}, T x_{n_{i}}\right) \geq \varepsilon \text { and } d\left(T x_{m_{i}}, T x_{n_{i}-1}\right)<\varepsilon
\end{aligned}
$$

So that $\quad \varepsilon \leq d\left(T x_{m_{i}}, T x_{n_{i}}\right) \leq d\left(T x_{m_{i}}, T x_{n_{i}-1}\right)+d\left(T x_{n_{i}-1}, T x_{n_{i}}\right)<\varepsilon+d\left(T x_{n_{i}-1}, T x_{n_{i}}\right)$

Therefore $\lim _{i \rightarrow \infty} d\left(T x_{m_{i}}, T x_{n_{i}}\right)=\varepsilon$

$$
\begin{gathered}
\text { Now } d\left(T x_{m_{i}}, T x_{n_{i}}\right) \leq d\left(T x_{m_{i}}, T x_{m_{i}-1}\right)+d\left(T x_{m_{i}-1}, T x_{n_{i}-1}\right)+d\left(T x_{n_{i}-1}, T x_{n_{i}}\right) \\
d\left(T x_{m_{i}-1}, T x_{n_{i}-1}\right) \leq d\left(T x_{m_{i}-1}, T x_{m_{i}}\right)+d\left(T x_{m_{i}}, T x_{n_{i}}\right)+d\left(T x_{n_{i}}, T x_{n_{i}-1}\right)
\end{gathered}
$$

By taking limit as $i \rightarrow \infty$, we get $\lim _{i \rightarrow \infty} d\left(T x_{m_{i}-1}, T x_{n_{i}-1}\right)=\varepsilon$

Now by (iii) and (3)

$$
\varphi(\varepsilon) \leq \varphi\left(d\left(T x_{m_{i}}, T x_{n_{i}}\right)\right) \leq \varphi\left(M\left(x_{m_{i}}, x_{n_{i}}\right)\right)-\phi\left(M\left(x_{m_{i}}, x_{n_{i}}\right)\right)
$$

where $M\left(x_{m_{i}}, x_{n_{i}}\right)=F\left\{d\left(T x_{m_{i}}, f x_{n_{i}}\right), d\left(T x_{n_{i}}, f x_{m_{i}}\right), d\left(T x_{m_{i}}, f x_{m_{i}}\right), d\left(T x_{n_{i}}, f x_{n_{i}}\right)\right\}$

$$
=F\left\{d\left(T x_{m_{i}}, T x_{n_{i}-1}\right), d\left(T x_{n_{i}}, T x_{m_{i}-1}\right), d\left(T x_{m_{i}}, T x_{m_{i}-1}\right), d\left(T x_{n_{i}}, T x_{n_{i}-1}\right)\right\}
$$

By taking limit as $i \rightarrow \infty$, we get $\lim _{i \rightarrow \infty} M\left(x_{m_{i}}, x_{n_{i}}\right)=F(\varepsilon, \varepsilon, 0,0)$

Thus form $\left(F_{3}\right)$ we have, $\lim _{i \rightarrow \infty} M\left(x_{m_{i}}, x_{n_{i}}\right) \leq \varepsilon$

Therefore $\quad \varphi(\varepsilon) \leq \varphi(\varepsilon)-\phi(\varepsilon)$ and hence $\phi(\varepsilon) \leq 0$ 
This is a contradiction because as $\phi:[0, \infty) \rightarrow[0, \infty)$, we must have $\phi(\varepsilon) \geq 0$ and $\phi(\varepsilon)=0$ if and only if $\varepsilon=0$ but $\varepsilon>0$.

Hence our supposition is false.

Thus $\left\{T x_{n}\right\}$ is a Cauchy sequence in $T(X)$.

But by (ii), $T(X)$ is a complete subset of $X$, there exists a point $q$ in $X$ such that

$$
\lim _{n \rightarrow \infty} T x_{n}=q
$$

Since $T(X) \subseteq f(X)$, there exists a point $p \in X$ such that $q=f p$.

Now from (iii), $\quad \varphi\left(d\left(T x_{n}, T p\right)\right) \leq \varphi\left(M\left(x_{n}, p\right)\right)-\phi\left(M\left(x_{n}, p\right)\right)$

where $M\left(x_{n}, p\right)=F\left\{d\left(T x_{n}, f p\right), d\left(T p, f x_{n}\right), d\left(T x_{n}, f x_{n}\right), d(T p, f p)\right\}$

$$
\begin{aligned}
& =F\left\{d\left(T x_{n}, f p\right), d\left(T p, T x_{n-1}\right), d\left(T x_{n}, T x_{n-1}\right), d(T p, f p)\right\} \\
& =F\{d(q, q), d(T p, q), d(q, q), d(T p, q)\} \text { (By taking limit as } n \rightarrow \infty) \\
& =F\{0, d(T p, q), 0, d(T p, q)\}
\end{aligned}
$$

Thus form $\left(\mathrm{F}_{3}\right)$ we have, $M\left(x_{n}, p\right) \leq d(T p, q)$

Therefore $\varphi(d(q, T p)) \leq \varphi(d(T p, q))-\phi(d(T p, q))$

Which implies that $\phi(d(T p, q)) \leq 0$ and this is possible only if $T p=q$.

Thus $T p=q=f p$ and hence $p$ is the coincidence point of $T$ and $f$.

Since $T$ and $f$ are weakly compatible, they commute at their coincidence point.

i.e., $T f p=f T p$ which implies that $T q=f q$.

Again from (iii), $\varphi(d(T q, T p)) \leq \varphi(M(q, p))-\phi(M(q, p))$

Where $M(q, p)=F\{d(T q, f p), d(T p, f q), d(T q, f q), d(T p, f p)\}$

$$
\begin{aligned}
& =F\{d(T q, q), d(q, T q), d(T q, T q), d(q, q)\} \\
& =F\{d(T q, q), d(q, T q), 0,0\}
\end{aligned}
$$

Thus form $\left(F_{3}\right)$ we have, $M(q, p) \leq d(T q, q)$

Therefore $\varphi(d(q, T q)) \leq \varphi(d(T q, q))-\phi(d(T q, q))$

Which implies that $\phi(d(T q, q)) \leq 0$ and this is possible only if $T q=q$.

Thus $T q=q=f q$ and hence $q$ is the common fixed point of $T$ and $f$.

Uniqueness: $\quad$ For uniqueness of $q$ let if possible, we assume that $q$ and $t,(q \neq t)$ are common fixed points of $f$ and $T$

from (iii), $\quad \varphi(d(T q, T t)) \leq \varphi(M(q, t))-\phi(M(q, t))$

where $M(q, t)=F\{d(T q, f t), d(T t, f q), d(T q, f q), d(T t, f t)\}$

$$
=F\{d(q, t), d(q, t), 0,0\}
$$

Thus form $\left(F_{3}\right)$ we have, $M(q, t) \leq d(q, t)$

Therefore $\varphi(d(q, t)) \leq \varphi(d(q, t))-\phi(d(q, t))$ 


\section{Fixed Point for Weakly Compatible Maps}

Which implies that $\phi(d(q, t)) \leq 0$ and this is possible only if $q=t$.

Hence the theorem.

\section{Application as best approximation}

Definition 3.1 Let $M$ be a nonempty subset of a metric space $(X, d)$. The set of best $M$ approximants to $u \in X$, denoted as $P_{M}(u)$ is defined by

$$
P_{M}(u)=\{y \in M: d(y, u)=d \dot{s} t(u, M)\}
$$

where $d \dot{s} t(u, M)=\inf \{d(x, u): x \in M\}$.

Theorem 3.2 Let $T$ and $f$ be self mappings of a metric space $(X, d)$. Suppose that $u \in X, T$ and $f$ satisfy following condition

$$
\varphi(d(T x, T y)) \leq \varphi(M(x, y))-\phi(M(x, y))
$$

where $M(x, y)=F\{d(T x, f y), d(T y, f x), d(T x, f x), d(T y, f y)\}$ and

$\varphi, \phi:[0, \infty) \rightarrow[0, \infty)$ are both monotonic increasing function with $\varphi(x)=0=\phi(x)$ if and only if $x=0$.

$T$ leaves $f$-invariant compact subset $M$ of closed subspace $f(X)$ as invariant. For each $b \in P_{M}(u)$, let $d(x, T b)<f(x, f b)$ and $f b \in P_{M}(u)$. If $T$ and $f$ are weakly compatible, then $u$ has a best approximation in $M$ which is also a common fixed point of $T$ and $f$.

Proof: Let $u \in F(T) \cap F(f)$

Since $M$ is a compact subset of $f(X), P_{M}(u) \neq \phi$.

We claim that $T\left(P_{M}(u)\right) \subseteq f\left(P_{M}(u)\right)$,

Let if possible we assume that, there exists $b \in P_{M}(u)$ such that $T b \notin f\left(P_{M}(u)\right)$.

Now $\quad d(u, f b)=\operatorname{dist}(u, M) \leq d(u, T b)<d(u, f b)$ which is a contradiction.

Hence $T\left(P_{M}(u)\right) \subseteq f\left(P_{M}(u)\right)$.

Now $f\left(P_{M}(u)\right)$ being a closed subset of a complete space is complete.

Hence $P_{M}(u) \cap F(T) \cap F(f)$ is singleton.

\section{Acknowledgement}

We are thankful to Prof. L. N. Joshi and Prof. J. N. Chauhan for their cooperation in the preparation of this paper.

\section{References}

1. S. Sessa, Publ. Inst. Math. (N.S.) 32 (46), 149 (1982). 
2. G. Jungck, Internet J.Math. Sci. 9, 43 (1986).

3. R. P. Pant, J. Math. Anal. Appl. 188, 436 (1994). http://dx.doi.org/10.1006/jmaa.1994.1437

4. G. Jungck and B. E. Rhoades, Indian J. Pure. Appl. Math. 29 (3), 227 (1998).

5. I. Beg and M. Abbas, Fixed point theory and Appl. article ID 74503, 1 (2006).

6. P. N. Dutta and B. S. Choudhury, Fixed point theory and Appl. article ID406368, 1 (2008).

7. B. E. Rhoades, Nonlinear Analysis: Theory, Methods and Applications, 47 (4), 2683 (2001).

8. K. P. R. Sastry and G. V. R. Babu, Indian J. pure appl. Math. 30 (6), 641 (1999).

9. T. Suzuki, Proc. Amer. Math. Soc. 136 (5), 1861 (2008). http://dx.doi.org/10.1090/S0002-9939-07-09055-7

10. S. Park, J. Korean Math. Soc. 16 (2), 95 (1980).

11. M. S. Khan, M. Swaleh, and S. Sessa, Bull. Austral. Math. Soc. 30, 1 (1984). http://dx.doi.org/10.1017/S0004972700001659

12. G. Jungck, Amer. Math. Monthly 83, 261 (1976). http://dx.doi.org/10.2307/2318216

13. G. Meinardus, Arch. Rational Mech. Anal. 14, 301 (1963).

14. B. Brosowski, Mathematica (Cluj) 11, 195 (1969).

15. P. V. Subrahmanyam, J. Approx. Theory 20, 165 (1977). http://dx.doi.org/10.1016/0021-9045(77)90070-3

16. S. P. Singh, J. Approx. Theory 25, 89 (1979). http://dx.doi.org/10.1016/0021-9045(79)90036-4

17. T. L. Hicks and M. D. Humphries, J. Approx. Theory 34, 221 (1982). http://dx.doi.org/10.1016/0021-9045(82)90012-0

18. L. Habiniak, J. Approx. Theory 56 , 241 (1989). http://dx.doi.org/10.1016/0021-9045(89)90113-5

19. G. Jungck and S. Sessa, Math. Japan 42 (2), 249 (1995).

20. S. A. Sahab, M. S. Khan and S. Sessa, J. Approx. Theory 55, 349 (1988). http://dx.doi.org/10.1016/0021-9045(88)90101-3

21. N. Shahzad, J. Math. Anal. Appl. 257, 39 (2001). http://dx.doi.org/10.1006/jmaa.2000.7274 